\title{
Epithelial Nuclear Factor-kB Activation in Inflammatory Bowel Diseases and Colitis-Associated Carcinogenesis
}

\author{
Takashi Nagaishi Taro Watabe Nisha Jose Arisa Tokai Toshimitsu Fujii \\ Katsuyoshi Matsuoka Masakazu Nagahori Kazuo Ohtsuka \\ Mamoru Watanabe
}

Department of Gastroenterology, Graduate School of Medical Science, Tokyo Medical and Dental University, Tokyo, Japan

\section{Key Words}

Colitis-associated cancer - Inflammatory bowel diseases .

Myosin light chain kinase $\cdot$ Tight junction · Tumor necrosis factor

\begin{abstract}
Prolonged inflammatory bowel diseases (IBD) may lead to colitis-associated carcinogenesis (CAC). Previous studies had shown that nuclear factor-KB (NF-KB) activation in both macrophages and epithelia in inflamed colonic tissue is associated with CAC development. However, the mechanism by which epithelial NF-KB activation leading to CAC development had not previously been rigorously studied. We and others had observed the increased expression of the type 2 receptor for tumor necrosis factor (TNFR2/TNFRSF1b/p75) in IBD models. Myosin light chain kinase (MLCK) is suggested to be associated with epithelial permeability via TNF signaling. Therefore, the relationship between epithelial MLCK expression and NF-KB activation via TNFR2 signaling on CAC development was investigated. Pro-tumorigenic cytokines such as interleukin (IL)-1 $\beta$, IL-6 and macrophage inflamma-
\end{abstract}

tory protein-2 at the lamina propria were increased in the setting of colitis and further increased in tumor tissues with upregulated epithelial TNFR2 and MLCK expressions in an animal model of CAC. The upregulated MLCK expression was also observed in TNF-stimulated colonic epithelial cells in vitro in association with the upregulation of TNFR2 but not TNFR1/TNFRSF1a/p55. Gene silencing of tnfrsf1 b, but not tnfrsf1a, resulted in restoration of epithelial tight junction (TJ) associated with decreased MLCK expression. The presence of anti-TNF antibody also resulted in restoration of TJ in association with suppressed MLCK expression, and interestingly, similar results including the suppressed TNFR2 and MLCK expressions were observed by inhibiting MLCK in the epithelial cells. MLCK silencing also led to suppressed TNFR2 expression, suggesting that the restored TJ leads to reduced TNFR2 signaling. Such suppression of MLCK as well as blockade of TNFR2 signaling resulted in reduced CAC development, restored TJ, and decreased pro-tumorigenic cytokines. These imply that TNF-induced NF-KB activation and MLCK expression may be a potential target for the prevention of IBD-associated carcinogenesis.

(c) 2016 S. Karger AG, Basel

\section{KARGER}

E-Mail karger@karger.com www.karger.com/dig
(C) 2016 S. Karger AG, Basel

$0012-2823 / 16 / 0931-0040 \$ 39.50 / 0$
Takashi Nagaishi, MD, PhD

Department of Gastroenterology and Hepatology Graduate School of Medical Science, Tokyo Medical and Dental University 1-5-45 Yushima, Bunkyo-ku, Tokyo 113-8519 (Japan)

E-Mail tnagaishi.gast@tmd.ac.jp 


\section{Introduction}

Inflammatory bowel diseases (IBD) such as ulcerative colitis (UC) and Crohn's disease (CD) in humans may cause idiopathic and chronic inflammation in colorectal area, as well as the entire gastrointestinal tract [1-3]. Although the etiology of IBD is still unclear, chronic epithelial hyper-permeability seems to be one of the mechanisms by which extensive inflammatory factors infiltrate the irritated intestinal tissues $[4,5]$. Therefore, it is believed that induction of mucosal healing at the early stage is critical in the management of IBD [6,7]. Moreover, chronic inflammation is generally believed to promote carcinogenesis, and prolonged duration of IBD likely also leads to increased risk of colitis-associated cancer (CAC) in the inflamed epithelia [8-11].

A previous study using tissue-specific deletion models of the I $\mathrm{K} B$ kinase $\beta$ had shown that activation of nuclear factor- $\kappa \mathrm{B}(\mathrm{NF}-\kappa \mathrm{B})$ in the inflamed tissue is strongly associated with carcinogenesis [12]. In this regard, we have investigated the mechanism of NF- $\kappa \mathrm{B}$ activation in the colonic epithelial cells using a murine model of IBD. We have previously reported that increased expression of tumor necrosis factor (TNF) in a murine model of IBD is critical for the development of CAC [13]. TNF is known to be a pivotal cytokine associated with continuous immune dysregulation in the inflamed tissue of IBD $[14,15]$. But how does this cytokine affect the intestinal epithelial cells in IBD? In our previous study, specific upregulation of the type 2 receptor for TNF (TNFR2/ TNFRSF1b/p75) was observed in the inflamed intestinal epithelial cells [13]. This observation seems logical since TNFR2 can also activate NF- $\kappa B$, but it lacks the association with the death domains (DD) like that of TNFR1/TNFRSF1a/p55. However, the specific role of such NF- $\kappa \mathrm{B}$ activation in the inflamed epithelia via TNFR2 signaling in the context of CAC has not been elucidated.

On the other hand, it has been reported that myosin light chain (MLC) kinase (MLCK) is also expressed in the human intestinal tissues with IBD [16]. MLCK is classically known to be required for the contraction of actomyosin via the phosphorylation of MLC [17]. It is also essential to the permeability of epithelial barrier according to in vitro and in vivo studies and is associated with the production of pro-inflammatory cytokine, such as TNF, in the inflamed intestinal tissues $[16,18]$. The role of MLCK in the development of CAC is still unknown. It seems that one of the roles of epithelial NF- $\kappa B$ activation would be the induction of MLCK in the context of IBD.
Here, the relationship between epithelial MLCK expression and NF- $\kappa B$ activation via TNF signaling in the development of IBD-associated carcinogenesis is described.

\section{Pro-Inflammatory and Pro-Tumorigenic Cytokine Productions in IBD}

The pathogenesis of IBD is considered to be associated with both epithelial hyper-permeability and dysregulated immune responses to luminal contents, which include antigens derived from commensal bacteria in the gut. Regarding immune dysregulation, in patients with CD for example, an excessive amount of Th1 and Th17 cytokines, such as interferon (IFN)- $\gamma$ and interleukin (IL)-17 respectively, are secreted predominantly by the infiltrating $\mathrm{CD} 4^{+}$effecter T cells in the intestinal lamina propria $[6,19-21]$. On the other hand, Th2 cytokines such as IL-4, IL- 5 and IL-13 are produced by effecter T cells and invariant NKT cells, as well as Th1 cytokines in the patients with UC $[4,22]$. TNF, a proinflammatory cytokine, which is produced not only by such dysregulated effecter $\mathrm{T}$ cells but also by macrophages and granulocytes infiltrating the inflamed intestinal tissues, is involved in the dysregulated adoptive immune responses in CD and possibly UC as well. Current therapeutic approaches in neutralizing TNF using chimeric or humanized antibodies have provided some effective therapies in the management of $\operatorname{CD}[6,23,24]$ and to some extent UC as well [25]. Our efforts in the study of pathogenesis of IBD have shown that TNF is expressed mainly by inflammatory macrophages rather than effecter T cells in the dextran sodium sulfate (DSS) colitis model [13].

It is known that myeloid cell-derived macrophages and granulocytes are also capable of expressing other cytokines such as IL- $1 \beta$, IL- 6 and macrophage inflammatory protein (MIP)-2, a homolog of IL-8 in humans. These cytokines can induce pro-tumorigenic activities such as angiogenesis and tumor proliferation [26-29], but at the same time, these cytokines are also expressed by intestinal epithelial cells [30-33]. In fact, we have observed in previous and recent studies that the expression levels of these cytokines in colonic tissues are upregulated in DSS colitis and further upregulated in tumors [13,34]. CAC development was suppressed by the treatment with either MP6-XT22, an anti-TNF monoclonal antibodies, or ML-7, an inhibitor against to MLCK, in association with reduced cytokine productions mentioned above (fig. 1a). However, cytokine expressions from the isolated 

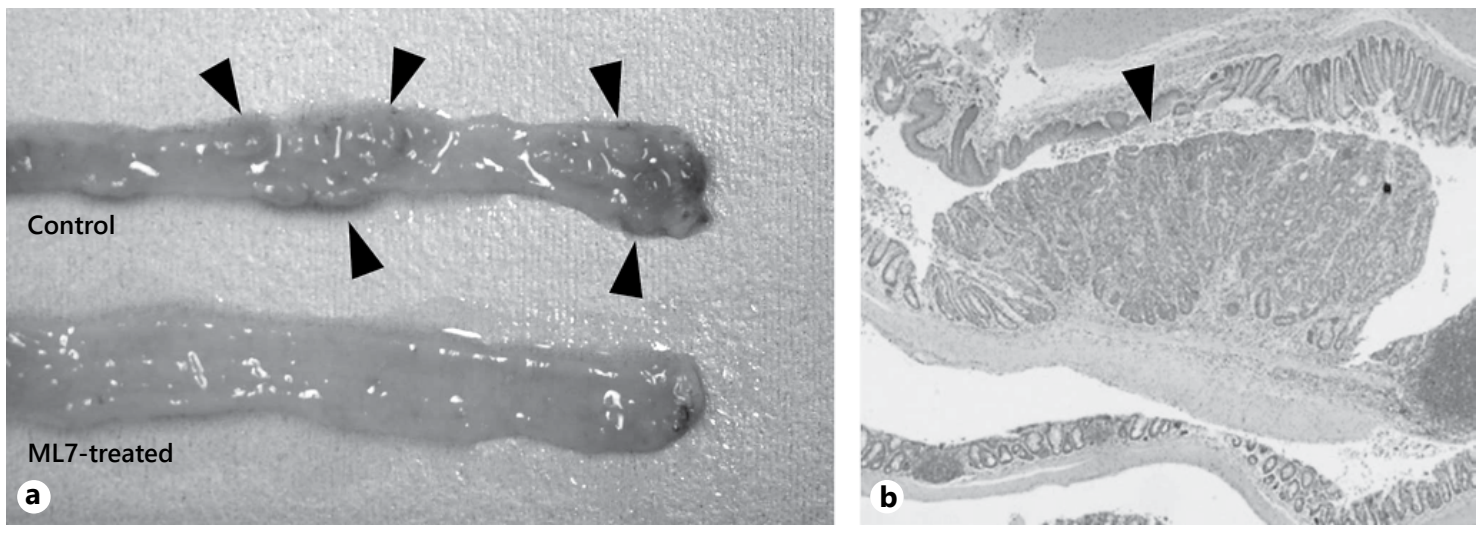

Fig. 1. Macroscopic and microscopic morphologies of CAC. a Representative macroscopic overviews of distal colon from AOM/DSS-administered mice with either vehicle control (control) or ML-7 treatment (ML7-treated) are shown. Arrow heads indicate colonic tumors in AOM/DSS-administered mice. b A rep- resentative histology of CAC from AOM/DSS-administered mice with vehicle control is shown. Sections were fixed with $10 \%$ neutral-buffered formalin and stained with hematoxylin and eosin. The arrow head indicates one of the tumors in mice. colonic epithelial cells were not significant when compared to those of the entire colonic mucosa. Therefore, pro-tumorigenic cytokines are most likely derived from the lamina propria where most macrophages reside. Others had also shown that NF- $\kappa B$ activation in the myeloid cells is critical for the induction of CAC [12], and that NF- $\kappa \mathrm{B}$ activation in myeloid cells can be induced by TNF [35].

\section{The Expressions of TNF Receptors in IBD and CAC}

DSS colitis is a commonly utilized murine model of IBD where a single administration of DSS can lead to acute colitis. In addition, 3 cycles of DSS administration can lead to chronic inflammation in the colon. Furthermore, azoxymethane (AOM) administration preceding 3 cycles of DSS treatment can lead to epithelial carcinogenesis and is a model of CAC (fig. la and b) [36]. By using this CAC model, Greten et al. [12] showed that epithelial NF- $\mathrm{KB}$ activation is critical for the development of CAC. However, the roles and mechanisms of epithelial NF- $\kappa$ B activation leading to CAC development had not previously been rigorously studied. In our previous and recent studies, we determined epithelial NF- $\mathrm{kB}$ activation in association with upregulated TNF production by macrophages infiltrating the colonic lamina propria in the same CAC model. In addition, we demonstrated that epithelial NF- $\kappa B$ activation was abrogated by neutralizing TNF with a specific monoclonal antibodies leading to the suppression of CAC $[13,34]$. These results suggest that the NF- $\kappa \mathrm{B}$ pathway in the epithelial cells is mainly activated by the upregulated TNF production. Previous studies by others had suggested that insufficient immunological homeostasis due to TNF production in IBD was caused by sequential activation of macrophages and neutrophils expressing TNFR1 in the inflamed tissue [35]. However, we and other researchers had observed that intestinal epithelial cells may express both TNFR1 and TNFR2 $[13,37]$. There is spontaneous TNFR2 upregulation in the epithelial cells in the setting of in vitro and in vivo inflammatory conditions $[13,38]$. An increased epithelial expression of TNFR2 was associated with CAC development [13]. These results suggest that TNF-induced epithelial NF- $\kappa \mathrm{B}$ activation is associated with CAC development.

Given the results of upregulated TNFR2 expression and NF- $\kappa B$ activation in the inflamed epithelial cells, we hypothesized that this may somehow be associated with reducing the recruitment of DDs, such as Fas-associated DD and TNFR1-associated DD, which are involved in the caspase-dependent pathway of TNFR1 signaling, so that the epithelial cells may escape apoptosis. However, the role of the epithelial NF- $\kappa B$ activation via TNFR2 signaling associated with CAC induction was not known previously. We therefore investigated whether an increased expression of TNFR2 in this model leads to CAC. Using the same model with AOM and DSS, we have tried to induce CAC in TNFR2-deficient mice. However, CAC development was not possible due to high mortality with DSS treatment, which had also been observed previously [13]. Therefore, we have utilized aMoC1, which is a murine 

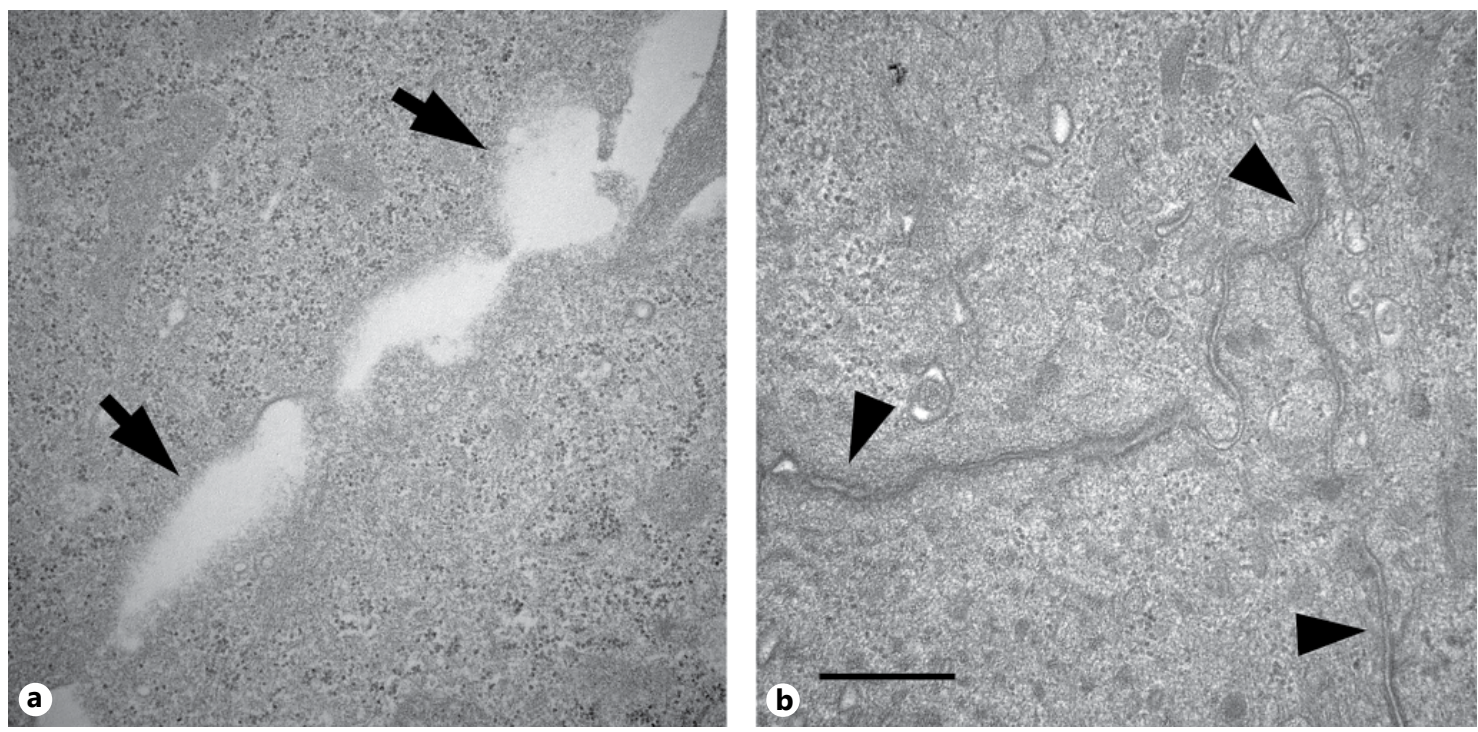

Fig. 2. TEM images of TJ in aMoC1 cells. a Disruption of intercellular junctional complexes. Arrows indicate disrupted intercellular junctional complex of aMoC1 cells with rIFN- $\gamma$ and rTNF stimulation. b Abrogation of disrupted junctional complexes. Arrow heads indicate fine intercellular junctional complexes of aMoC1 cells in the presence of ML-7. Representative TEM images are shown. Scale bar indicates $500 \mathrm{~nm}$. epithelial cell line derived from non-cancerous colonic epithelia transformed with SV40 large T antigen [39]. This cell line likely possesses more physiological functions compared to other cell lines derived from murine colon cancer such as CT26 cells [40]. Furthermore, aMoC1 cells can form confluent monolayers with epithelial tight junctions (TJ). Such characters of aMoC1 cells allowed us to assess the collapsed TJ associated with MLCK upregulation, because it was difficult to observe such morphologies with CT26 cells, which does not show either MLCK upregulation or fine monolayer required for the observations of intercellular junctions under confocal and transmission electron microscopies (TEM; fig. 2).

\section{TNF-Induced Epithelial Hyper-Permeability and CAC Development}

It had been shown that MLCK in human intestinal cells leads to TNF-induced collapse of epithelial TJ via TNFR2 signaling [41] and that MLCK promoter activity is mediated by NF- $\kappa B$ [42-44]. Therefore, TNFR2 expression was silenced in aMoC1 cells by transfection with the specific small interfering RNA to determine MLCK activities in the presence of recombinant (r) TNF in our study [34]. This resulted in the abrogation of TNF- induced TJ disruption associated with suppressed MLCK upregulation, and these results are consistent with previous reports by others $[18,41]$. Moreover, we also observed that the TJ remained intact with anti-TNF treatment as well as inhibiting MLCK activity (fig. $2 a$ and b). These findings may imply an important mechanism by which NF- $\kappa$ B pathway is regulated in the colonic epithelial cells in the setting of carcinogenesis. Thus, one potential mechanism by which TNFR2 expression in intestinal epithelial cells is associated with CAC development may be that the epithelial barrier dysfunction induced by MLCK activation via TNFR2 signaling leads to translocation of luminal bacteria into the lamina propria. Such epithelial hyper-permeability results in the stimulation of infiltrating macrophages and granulocytes in mucosal tissue followed by cytokine production such as IL- $1 \beta$, IL- 6 and MIP- 2 that are required for epithelial proliferation and tumor development (fig. 3). It should be noted that the impact of such pro-tumorigenic cytokines production, which is induced by disrupted TJ, on the severity of DSS colitis is controversial. Some recent studies have suggested that suppression of epithelial permeability by the administration of MLCK inhibitors ameliorates DSS colitis $[45,46]$. However, our recent studies suggest that suppression of colitis by ML-7 was not significant, although such treatment was remarkably effective on the suppression of CAC development. One rea- 


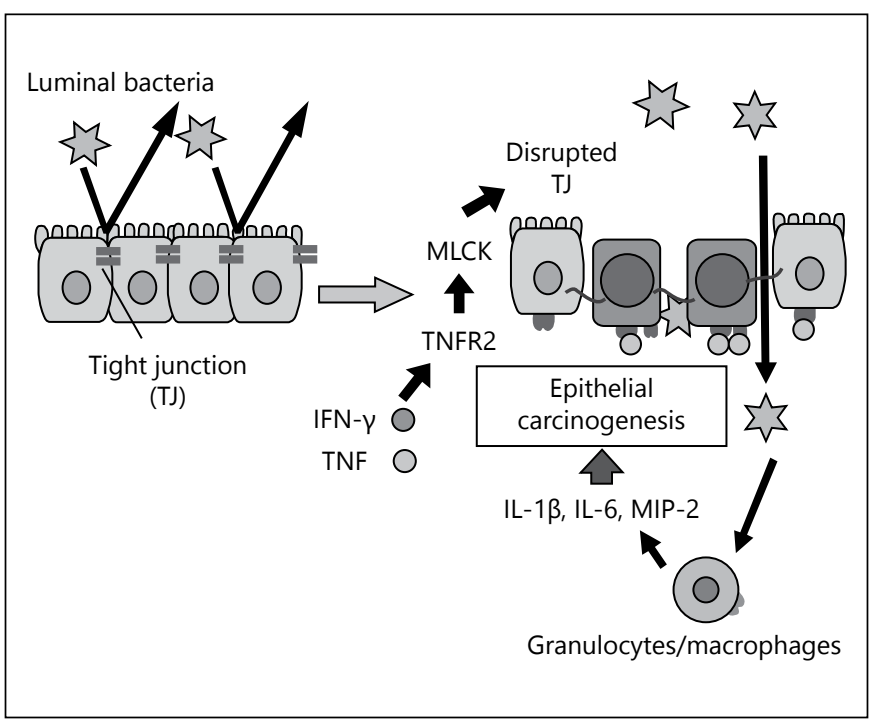

Fig. 3. Mechanism of TNF-induced CAC development.

son for the different observations may be that the severity of colitis in our studies was relatively mild compared to the others, and thus, we did not observe distinct abrogation of colitis by MLCK inhibition. In addition, another recent report showed that MLCK-deficient mice revealed worse DSS colitis [47]. These discrepant observations by others and ours imply that another reason may be contributed by environmental factors such as the different microflora at the animal facilities. In humans, on the other hand, it is known that the epithelial NF-kB is activated in IBD, and TNF is usually a critical cytokine in many cases of CD and UC pathogenesis. These suggest that the MLCK expression is presumably one of key molecules that affect the activity and duration of inflammation in human IBD.

In our recent study, rINF- $\gamma$ and rTNF-induced upregulation of TNFR2, and possibly MLCK, in the epithelial cells were suppressed by ML-7 treatment [34]. Upregulation of TNFR2 was also suppressed by silencing MLCK expression. These results suggest that TJ disruption, which is induced by MLCK expression and MLC phosphorylation in the epithelial cells, may result in further upregulation of TNFR2 expression. This in turn causes a vicious circle on the epithelial hyper-permeability, and such a condition may promote tumor growth in the context of inflammation. In this respect, we have examined whether such MLCK expression also induces upregulation of endogenous IFN- $\gamma$ and/or TNF expressions in these cells. Neither expression of these was affected regardless of the MLCK expression (unpublished). There- fore, another mechanism seems to be involved in the TNFR2 upregulation in the irritated epithelial cells. Furthermore, these data suggest that MLCK expression, which may be induced by TNFR2 signaling in the intestinal epithelial cells, can be a therapeutic target for the maintenance of continuous inflammation and prevention of CAC development in the setting of IBD.

It cannot be exactly determined whether the NF- $\kappa \mathrm{B}$ activation through TNFR2 signaling and MLCK expression would be directly responsible for the induction of epithelial dysplasia, since we did not go through the entire tissue to check for aberrant crypt foci. However, it should be noted that we have observed that both MP6-XT22 and ML-7 treatments resulted in the decrease of microscopic tumors as well as larger tumors $[13,34]$. These results imply that the expression of MLCK may be associated with not only tumor growth but also epithelial cell dysplasia in the context of IBD. It is already known that p53, Wnt and EGFR signaling are involved in colorectal carcinogenesis. In this regard, neither TNFR2 signaling nor MLCK expression has been reported to be associated with these signaling above.

\section{Conclusion}

We described TNFR2-mediated epithelial NF- $\kappa \mathrm{B}$ activation and CAC development, which is due to tumorigenic cytokines induced by MLCK-induced disruption of the TJ. This is an important mechanism that helps us understand the mechanisms of extensive cytokine production via chronic epithelial hyper-permeability in IBD, as well as IBD-associated carcinogenesis.

\section{Acknowledgments}

This study was supported in part by Grants-in-Aid for Scientific Research (T.N. and M.W.); Scientific Research on Priority Areas (M.W.); Exploratory Research and Creative Scientific Research (T.N.) from the Japanese Ministry of Education, Culture, Sports, Science and Technology; the Japanese Ministry of Health, Labor and Welfare (M.W.); the Japan Medical Association (M.W.); Otsuka Toshimi Scholarship Foundation (N.J.); Memorial Fund of Nihon University Medical Alumni Association (T.N.); Abbott Japan Allergy Research Award (T.N.); Foundation for Advancement of International Science (T.N.); Takeda Science Foundation (T.N.).

\section{Disclosure Statement}

The authors have no financial conflict of interest.
44

Digestion 2016;93:40-46 DOI: $10.1159 / 000441670$
Nagaishi/Watabe/Jose/Tokai/Fujii/ Matsuoka/Nagahori/Ohtsuka/Watanabe 


\section{References}

1 Ueno F, Matsui T, Matsumoto T, Matsuoka K, Watanabe M, Hibi T: Evidence-based clinical practice guidelines for Crohn's disease, integrated with formal consensus of experts in Japan. J Gastroenterol 2013;48:31-72.

2 Fujii T, Naganuma M, Kitazume Y, Saito E, Nagahori M, Ohtsuka K, Watanabe M: Advancing magnetic resonance imaging in Crohn's disease. Digestion 2014;89:24-30.

3 Saito E, Nagahori M, Fujii T, Ohtsuka K, Watanabe M: Efficacy of salvage therapy and its effect on operative outcomes in patients with ulcerative colitis. Digestion 2014;89:5560.

4 Xavier RJ, Podolsky DK: Unravelling the pathogenesis of inflammatory bowel disease. Nature 2007;448:427-434.

5 Langhorst J, Lauche R: It is about time - tailoring of an individualized multimodal treatment approach in ulcerative colitis. Digestion 2014;89:139-141.

6 Hanauer SB, Feagan BG, Lichtenstein GR, Mayer LF, Schreiber S, et al: Maintenance infliximab for Crohn's disease: the ACCENT I randomised trial. Lancet 2002;359:15411549.

7 Sakuraba H, Ishiguro Y, Hasui K, Hiraga H, Fukuda S, Shibutani K, Takai Y: Prediction of maintained mucosal healing in patients with Crohn's disease under treatment with infliximab using diffusion-weighted magnetic resonance imaging. Digestion 2014;89: 49-54.

8 Ekbom A, Helmick C, Zack M, Adami HO: Ulcerative colitis and colorectal cancer. A population-based study. N Engl J Med 1990; 323:1228-1233.

9 Ekbom A, Helmick C, Zack M, Adami HO: Increased risk of large-bowel cancer in Crohn's disease with colonic involvement. Lancet 1990;336:357-359.

10 Ekbom A, Helmick CG, Zack M, Holmberg L, Adami HO: Survival and causes of death in patients with inflammatory bowel disease: a population-based study. Gastroenterology 1992;103:954-960.

11 Wang YR, Cangemi JR, Loftus EV Jr, Picco MF: Use of surveillance colonoscopy in medicare patients with inflammatory bowel disease prior to colorectal cancer diagnosis. Digestion 2014;90:58-62.

12 Greten FR, Eckmann L, Greten TF, Park JM, $\mathrm{Li} \mathrm{ZW}$, et al: IKKbeta links inflammation and tumorigenesis in a mouse model of colitis-associated cancer. Cell 2004;118:285-296.

13 Onizawa M, Nagaishi T, Kanai T, Nagano K, Oshima S, et al: Signaling pathway via TNFalpha/NF-kappaB in intestinal epithelial cells may be directly involved in colitis-associated carcinogenesis. Am J Physiol Gastrointest Liver Physiol 2009;296:G850-G859.

14 Derkx B, Taminiau J, Radema S, Stronkhorst A, Wortel C, et al: Tumour-necrosis-factor antibody treatment in Crohn's disease. Lancet 1993;342:173-174.
15 Pullman WE, Elsbury S, Kobayashi M, Hapel AJ, Doe WF: Enhanced mucosal cytokine production in inflammatory bowel disease. Gastroenterology 1992;102:529-537.

16 Blair SA, Kane SV, Clayburgh DR, Turner JR: Epithelial myosin light chain kinase expression and activity are upregulated in inflammatory bowel disease. Lab Invest 2006;86: 191-201.

17 Ikebe M, Inagaki M, Kanamaru K, Hidaka H: Phosphorylation of smooth muscle myosin light chain kinase by $\mathrm{Ca} 2+$-activated, phospholipid-dependent protein kinase. J Biol Chem 1985;260:4547-4550.

18 Wang F, Graham WV, Wang Y, Witkowski ED, Schwarz BT, et al: Interferon-gamma and tumor necrosis factor-alpha synergize to induce intestinal epithelial barrier dysfunction by up-regulating myosin light chain kinase expression. Am J Pathol 2005;166:409-419.

19 Nagaishi T, Pao L, Lin SH, Iijima H, Kaser A, et al: SHP1 phosphatase-dependent T cell inhibition by CEACAM1 adhesion molecule isoforms. Immunity 2006;25:769-781.

20 Brand S: Crohn's disease: Th1, Th17 or both? The change of a paradigm: new immunological and genetic insights implicate Th17 cells in the pathogenesis of Crohn's disease. Gut 2009;58:1152-1167.

21 Targan SR, Hanauer SB, van Deventer SJ, Mayer L, Present DH, et al: A short-term study of chimeric monoclonal antibody cA2 to tumor necrosis factor alpha for Crohn's disease. Crohn's Disease cA2 Study Group. N Engl J Med 1997;337:1029-1035.

22 Rutgeerts P, Sandborn WJ, Feagan BG, Reinisch W, Olson A, et al: Infliximab for induction and maintenance therapy for ulcerative colitis. N Engl J Med 2005;353:2462-2476.

23 Driscoll KE, Hassenbein DG, Howard BW, Isfort RJ, Cody D, et al: Cloning, expression, and functional characterization of rat MIP-2: a neutrophil chemoattractant and epithelial cell mitogen. J Leukoc Biol 1995;58:359-364.

24 Koch AE, Polverini PJ, Kunkel SL, Harlow LA, DiPietro LA, et al: Interleukin-8 as a macrophage-derived mediator of angiogenesis. Science 1992;258:1798-1801.

25 Lahm H, Petral-Malec D, Yilmaz-Ceyhan A, Fischer JR, Lorenzoni M, et al: Growth stimulation of a human colorectal carcinoma cell line by interleukin- 1 and -6 and antagonistic effects of transforming growth factor beta 1 . Eur J Cancer 1992;28A:1894-1899.

26 Coussens LM, Werb Z: Inflammation and cancer. Nature 2002;420:860-867.

27 Eckmann L, Jung HC, Schürer-Maly C, Panja A, Morzycka-Wroblewska E, et al: Differential cytokine expression by human intestinal epithelial cell lines: regulated expression of interleukin 8. Gastroenterology 1993;105: 1689-1697.

28 Li Y, de Haar C, Chen M, Deuring J, Gerrits MM, et al: Disease-related expression of the IL6/STAT3/SOCS3 signalling pathway in ul- cerative colitis and ulcerative colitis-related carcinogenesis. Gut 2010;59:227-235.

29 Yamaji O, Nagaishi T, Totsuka T, Onizawa M, Suzuki M, et al: The development of colitogenic CD4(+) T cells is regulated by IL-7 in collaboration with NK cell function in a murine model of colitis. J Immunol 2012;188: 2524-2536.

30 Brozovic S, Nagaishi T, Yoshida M, Betz S, Salas A, et al: CD1d function is regulated by microsomal triglyceride transfer protein. Nat Med 2004;10:535-539.

31 Sands BE, Anderson FH, Bernstein CN, Chey WY, Feagan BG, et al: Infliximab maintenance therapy for fistulizing Crohn's disease. N Engl J Med 2004;350:876-885.

32 Ohno Y, Lee J, Fusunyan RD, MacDermott RP, Sanderson IR: Macrophage inflammatory protein-2: chromosomal regulation in rat small intestinal epithelial cells. Proc Natl Acad Sci U S A 1997;94:10279-10284.

33 Prakash R, Bharathi Raja S, Devaraj H, Devaraj SN: Up-regulation of MUC2 and IL- $1 \beta$ expression in human colonic epithelial cells by Shigella and its interaction with mucins. PLoS One 2011;6:e27046.

34 Suzuki M, Nagaishi T, Yamazaki M, Onizawa M, Watabe T, Sakamaki Y, Ichinose S, Totsuka M, Oshima S, Okamoto R, Shimonaka M, Yagita H, Nakamura T, Watanabe M: Myosin light chain kinase expression induced via tumor necrosis factor receptor 2 signaling in the epithelial cells regulates the development of colitis-associated carcinogenesis. PLoS One 2014;9:e88369.

35 Popivanova BK, Kitamura K, Wu Y, Kondo T, Kagaya $T$, et al: Blocking TNF-alpha in mice reduces colorectal carcinogenesis associated with chronic colitis. J Clin Invest 2008;118:560-570.

36 Tanaka T, Kohno H, Suzuki R, Yamada Y, Sugie $S$, et al: A novel inflammation-related mouse colon carcinogenesis model induced by azoxymethane and dextran sodium sulfate. Cancer Sci 2003;94:965-973.

37 Mizoguchi E, Hachiya Y, Kawada M, Nagatani $\mathrm{K}$, Ogawa $\mathrm{A}$, et al: TNF receptor type I-dependent activation of innate responses to reduce intestinal damage-associated mortality. Gastroenterology 2008;134:470-480.

38 Mizoguchi E, Mizoguchi A, Takedatsu H, Cario E, de Jong YP, et al: Role of tumor necrosis factor receptor 2 (TNFR2) in colonic epithelial hyperplasia and chronic intestinal inflammation in mice. Gastroenterology 2002;122:134-144.

39 Iwamoto T, Yamada K, Shimizu M, Totsuka M: Establishment of intestinal epithelial cell lines from adult mouse small and large intestinal crypts. Biosci Biotechnol Biochem 2011; 75:925-929.

40 Wang M, Bronte V, Chen PW, Gritz L, Panicali D, et al: Active immunotherapy of cancer with a nonreplicating recombinant fowlpox virus encoding a model tumor-associated antigen. J Immunol 1995;154:4685-4692. 
41 Wang F, Schwarz BT, Graham WV, Wang Y, $\mathrm{Su}$ L, et al: IFN-gamma-induced TNFR2 expression is required for TNF-dependent intestinal epithelial barrier dysfunction. Gastroenterology 2006;131:1153-1163.

42 Graham WV, Wang F, Clayburgh DR, Cheng JX, Yoon B, et al: Tumor necrosis factor-induced long myosin light chain kinase transcription is regulated by differentiation-dependent signaling events. Characterization of the human long myosin light chain kinase promoter. J Biol Chem 2006;281:2620526215.
43 Ye D, Ma TY: Cellular and molecular mechanisms that mediate basal and tumour necrosis factor-alpha-induced regulation of myosin light chain kinase gene activity. J Cell Mol Med 2008;12:1331-1346.

44 Ye D, Ma I, Ma TY: Molecular mechanism of tumor necrosis factor-alpha modulation of intestinal epithelial tight junction barrier. Am J Physiol Gastrointest Liver Physiol 2006; 290:G496-G504.
45 Chen C, Wang P, Su Q, Wang S, Wang F: Myosin light chain kinase mediates intestinal barrier disruption following burn injury. PLoS One 2012;7:e34946.

46 Liu X, Xu J, Mei Q, Han L, Huang J: Myosin light chain kinase inhibitor inhibits dextran sulfate sodium-induced colitis in mice. Dig Dis Sci 2013;58:107-114.

47 Su L, Nalle SC, Shen L, Turner ES, Singh G, et al: TNFR2 activates MLCK-dependent tight junction dysregulation to cause apoptosismediated barrier loss and experimental colitis. Gastroenterology 2013;145:407-415. 